\title{
Higher-order nonlinear electromechanical effects in wurtzite GaN/AIN quantum dots
}

\author{
Mehrdad Bahrami-Samani ${ }^{1}$, Sunil R Patil ${ }^{1,2}$ and \\ Roderick Melnik $^{1,3}$ \\ ${ }^{1} \mathrm{M}^{2} \mathrm{NeT}$ Laboratory, Wilfrid Laurier University, 75 University Avenue W, Waterloo, ON, \\ N2L 3C5, Canada \\ 2 Department of Physics, College of Engineering Pune, Pune 411005, India \\ ${ }^{3}$ BCAM Bizkaia Technology Park, E-48160 Derio, Spain \\ E-mail: mbahrami@wlu.ca, spatil@wlu.ca and rmelnik@wlu.ca
}

Received 8 September 2010

Published 23 November 2010

Online at stacks.iop.org/JPhysCM/22/495301

\begin{abstract}
As we demonstrated earlier, conventional mathematical models based on linear approximations may be inadequate in the analysis of properties of low-dimensional nanostructures and band structure calculations. In this work, a general three-dimensional axisymmetric coupled electromechanical model accounting for lattice mismatch, spontaneous polarization and higher-order nonlinear electrostriction effects has been applied to analyze properties of GaN/AlN quantum dots coupled with wetting layer. The generalized model that accounts for five independent electrostriction coefficients has been solved numerically via a finite-element implementation. The results, exemplified for truncated conical GaN/AlN quantum dots, demonstrate that the effect of nonlinear electrostriction in GaN/AlN nanoheterostructure quantum dots could be significant. In particular, the influence of nonlinear electromechanical effects on optoelectronic properties is highlighted by the results on band structure calculations based on a multiband effective mass theory.
\end{abstract}

(Some figures in this article are in colour only in the electronic version)

\section{Introduction}

GaN/AlN nanodimensional heterostructures are the promising candidates for current and potential applications in optoelectronics due to their unique properties $[1,2]$. The optoelectronic properties of the nanostructures are being engineered with strain and related effects [3-5]. These effects may also be coupled with a combination of thermal, mechanical and electromagnetic effects $[2,6]$, making the analysis of band structures of such nanostructures a very challenging task. While many such effects, including piezoelectric, can often be described with linear mathematical models, in some cases we need to deal with complex nonlinear phenomena associated with such couplings where linear models become inadequate [7-9]. An example of this is provided by phase transformations and other nonlinear phenomena where a combined influence of stress and temperature may substantially affect band structures and properties of corresponding materials $[10,11]$. The analysis of such nonlinear phenomena can efficiently be carried out with recently developed numerical methodologies and model reductions (see [12-14] and references therein).
GaN/AlN-based nanostructures belong to a group of nanostructures where coupled nonlinear effects and phenomena are expected to be of considerable interest [15, 16]. Indeed, recall that the static and dynamic influence of spontaneous polarization, piezoelectric effects, lattice mismatch and strain effects are significant in GaN/AlN wurtzite structures [17]. Furthermore, GaN has been found to possess a significant quadratic component in its electromechanical response [18]. Due to low symmetry of the wurtzite crystal lattice, the built-in piezoelectric field at the GaN/AlN interface governs optical properties and electrical polarization, and has a great impact on their optoelectronic properties [19-22]. Also, due to a number of effects that were accounted for in the past (e.g. those caused by a high built-in electric field in GaN/AlN and others) the conventional application of linear models to the analysis of optoelectromechanical properties of nanostructures in bandstructure engineering was inadequate $[9,23]$. These effects, which in many cases are nonlinear [11, 24-27], and their influence on optoelectronic properties of nanostructures deserve further and more detailed studies. 
A multi-dimensional self-consistent problem [6, 15] accounting for nonlinear electrostrictive effects has not yet been fully addressed in spite of the recent experimental and computational evidence demonstrating that a combination of dimensional effects and electrostriction could lead to the interesting new physics [28]. Here, we focus on the threedimensional axisymmetric case (effectively reduced to a $2 \mathrm{D}$ case due to symmetry), paying particular attention to the influence of higher-order nonlinear electromechanical effects in quantum dots (QDs).

Up to date, much research on electrostriction of lowdimensional nanostructures has been focused on single-walled carbon nanotubes (CNTs) [29, 30] and with a widening range of CNT applications [31] this research will continue to be vital. This is also true in the context of various types of electroactive polymers, elastomers, polycrystalline ceramics, ferroelectrics and electrostriction-based devices where some results on experimental measurements are available in the literature [32-34]. The importance of nonlinear electromechanical effects with their consequent influence on properties of such nanostructures as quantum dots have also been reported in the literature [28, 35-37]. However, no consistent systematic studies based on the fully nonlinear model accounting for electrostriction phenomena in lowdimensional nanostructures have been undertaken.

In what follows, we present a fully coupled electro-elastic, three-dimensional axisymmetric model accounting not only for lattice mismatch and spontaneous polarization, but also for higher-order nonlinear electrostrictive effects and apply this model to investigate the effect of electrostriction on properties of GaN/AIN nanoheterostructure QDs. Since the coupling between QDs and wetting layer (WL) is essential [38-41], we present the results for truncated conical QDs with WL as a representative example. The truncated conical QDs had a bottom radius, top radius and height of 9, 6 and $3 \mathrm{~nm}$, respectively, coupled with a $2 \mathrm{~nm}$ thick wetting layer, as an example. The dimensions used are close to the experimentally grown QDs [2].

\section{Theoretical formulation}

Our model is based on the coupled system of elasticity equations and Maxwell's equations with constitutive relationships in the axisymmetrical case accounting for the piezoelectric field [42]. Nonlinear constitutive equations for wurtzite crystal structures accounting for higher-order effects such as nonlinear stiffness, electrostrictive, elastostrictive and nonlinear dielectric permittivity are taken into account [43] and the corresponding constitutive relationships are used to construct the governing equations. Our coupled electro-elastic model takes into account all five non-zero independent electrostriction coefficients, while other higher-order nonlinear terms such as nonlinear stiffness, elastostrictive and dielectric permittivity coefficients are neglected. This allows us to single out the contribution of electrostriction on GaN/AIN quantum dot properties. The considered domain is discretized in triangular elements and the model is solved self-consistently via a finiteelement implementation. The resulting electromechanical distributions are then incorporated into the eight-band $\mathbf{k} \cdot \mathbf{p}$ model and subsequent band structure calculation is performed. We demonstrate that these contributions can be essential in explaining new physical properties of such low-dimensional nanostructures.

The coupling in our physics-based model is implemented via constitutive equations derived from the thermodynamic Gibb's potential as follows:

$$
\begin{aligned}
T_{i j}= & c_{i j l m}^{E} S_{l m}-e_{i j n} E_{n}+\frac{1}{2} c_{i j l m p q}^{E} S_{l m} S_{p q} \\
& -\frac{1}{2} B_{i j n r} E_{n} E_{r}-\gamma_{i j l m n} S_{l m} E_{n}, \\
D_{k}= & e_{k l m} S_{l m}+\epsilon_{k n}^{S} E_{n}+\frac{1}{2} \gamma_{k l m p q} S_{l m} S_{p q} \\
& +\frac{1}{2} \epsilon_{k n r}^{S} E_{n} E_{r}+B_{k l m n} S_{l m} E_{n}+P^{\mathrm{sp}},
\end{aligned}
$$

where the related parameters are stress $T_{i j}$, strain $S_{i j}$, electric field $E_{n}$, electric flux $D_{k}$ and spontaneous polarization $P^{\mathrm{sp}}$. The related linear coefficients in the above constitutive equations are referred to as the stiffness coefficient $c_{i j l m}^{E}=\left(\partial T_{i j} / \partial S_{l m}\right)_{E}$, dielectric permittivity $\epsilon_{k n}^{S}=\left(\partial D_{k} / \partial E_{n}\right)_{S}$ and piezoelectric coefficients $e_{i j n}=$ $\left(\partial T_{i j} / \partial E_{n}\right)_{S}=\left(\partial D_{k} / \partial S_{i j}\right)_{E}$. Higher-order nonlinear properties are defined here by the nonlinear stiffness coefficient $c_{i j l m p q}^{E}=\partial^{2} T_{i j} /\left(\partial S_{l m} \partial S_{p q}\right)$, nonlinear electrostriction coefficient $B_{i j n r}=\partial^{2} T_{i j} /\left(\partial E_{n} \partial E_{r}\right)=\partial^{2} D_{k} /\left(\partial S_{l m} \partial E_{n}\right)$, nonlinear electrostriction coefficient $\gamma_{i j l m n}=\partial^{2} T_{i j} /\left(\partial S_{l m} \partial E_{n}\right)=$ $\partial^{2} D_{k} /\left(\partial S_{l m} \partial S_{p q}\right)$ and nonlinear dielectric permittivity $\epsilon_{k n r}^{S}=$ $\partial^{2} D_{k} /\left(\partial E_{n} \partial E_{r}\right)$. In the following formulation, our major focus is on the nonlinear electrostriction coefficient $B_{i j n r}$.

The electrostriction coefficients, $B_{i j n r}$, are reduced for a piezoelectric poled in the $z$ direction which is also an axis of symmetry. The index reduction for the electrostriction coefficient in this case can be summarized as follows:

$$
\begin{gathered}
B_{122}=B_{1122}=B_{2211}, \\
B_{133}=B_{1133}=B_{2233}=B_{3311}=B_{3322}, \\
B_{423}=B_{2323}=B_{1313}, \\
B_{111}=B_{1111}=B_{2222}, \quad B_{333}=B_{3333} .
\end{gathered}
$$

Then, the corresponding term in equations (1) has the following form:

$$
\mathbf{B}=-\frac{1}{2}\left[\begin{array}{cccccc}
B_{111} & B_{122} & B_{133} & 0 & 0 & 0 \\
B_{122} & B_{111} & B_{133} & 0 & 0 & 0 \\
B_{133} & B_{133} & B_{333} & 0 & 0 & 0 \\
0 & 0 & 0 & B_{423} & 0 & 0 \\
0 & 0 & 0 & 0 & B_{423} & 0 \\
0 & 0 & 0 & 0 & 0 & \frac{\left(B_{111}-B_{122}\right)}{2}
\end{array}\right] \text {. }
$$

Before proceeding further, we note that we will analyze the influence of the electrostriction with electrostriction coefficient value ranging from $1.2 \times 10^{-18} \mathrm{~m}^{2} \mathrm{~V}^{-2}$ (reported in [18]) to $1.2 \times 10^{-21} \mathrm{~m}^{2} \mathrm{~V}^{-2}$. Indeed, the only known experimental electrostrictive coefficient [18] shows a relatively high value of electrostriction coefficient for GaN compared to the materials in the same group. Further, we use $M_{33}=$ $1.2 \times 10^{-18} \mathrm{~m}^{2} \mathrm{~V}^{-2}$ in strain terms, $B_{333}\left(\mathrm{C}^{2}\left(\mathrm{~N} \mathrm{~m}^{2}\right)^{-1}\right)=$ $-M_{33}\left(\mathrm{~m}^{2} \mathrm{~V}^{-2}\right) \times c_{33}^{E}(\mathrm{~Pa})$ (following notations of [44]) in 
stress terms [18]. We single out the coefficient $B_{333}$ due to the symmetry considerations and the fact that as far as we know it is the only experimental available coefficient. Indeed, the experimental studies of electrostriction properties of semiconductors are very limited. The authors of [18] measured the electrostrictive coefficient in the III-V semiconductor GaN by measuring the field-induced strain using an interferometric technique. We emphasize that the model presented here can account for all five independent coefficients in equations (2) and (3). However, for the reason indicated above we follow [18], focusing on the coefficient $B_{333}$.

The governing equations of coupled electromechanical interactions for the axisymmetrical case are reduced to the following two-dimensional system [45]:

$$
\begin{gathered}
\frac{\partial T_{r z}}{\partial r}+\frac{\partial T_{z z}}{\partial z}+\frac{T_{r z}}{r}=0, \\
\frac{\partial T_{r r}}{\partial r}+\frac{\partial T_{r z}}{\partial z}+\frac{T_{r r}-T_{\theta \theta}}{r}=0, \\
\frac{\partial D_{r}}{\partial r}+\frac{\partial D_{z}}{\partial z}+\frac{D_{r}}{r}=0 .
\end{gathered}
$$

Such coupled systems in their linear setting have been analyzed in detail mathematically [46-50, 45] with efficient numerical methodologies developed for their solution in $[51-53,45]$. The situation becomes even more challenging in the nonlinear case with no similar mathematically rigorous results known. Note also that, if transport phenomena need to be included in the analysis of nanostructures, additional effects may have to be included into the model (e.g. Pekar's mechanism for electron-phonon interactions and others [54]). We focus here on the time-independent situations. Given the above assumptions, the explicit form of nonlinear constitutive equations for wurtzite (GaN/AlN) crystal structures are

$$
\begin{aligned}
T_{r r}= & c_{11}^{E} S_{r r}+c_{12}^{E} S_{\theta \theta}+c_{13}^{E} S_{z z}-e_{31} E_{z} \\
& -\frac{1}{2} B_{111} E_{r}^{2}-\frac{1}{2} B_{133} E_{z}^{2}, \\
T_{\theta \theta}= & c_{12}^{E} S_{r r}+c_{11}^{E} S_{\theta \theta}+c_{13}^{E} S_{z z}-e_{31} E_{z} \\
& -\frac{1}{2} B_{122} E_{r}^{2}-\frac{1}{2} B_{133} E_{z}^{2}, \\
T_{z z}= & c_{13}^{E} S_{r r}+c_{13}^{E} S_{\theta \theta}+c_{33}^{E} S_{z z}-e_{33} E_{z} \\
& -\frac{1}{2} B_{133} E_{r}^{2}-\frac{1}{2} B_{333} E_{z}^{2}, \\
T_{r z}= & c_{44}^{E} S_{r z}-e_{15} E_{r}-\frac{1}{2} B_{423} E_{r} E_{z}, \\
D_{r}= & e_{15} S_{r z}+\epsilon_{11}^{S} E_{r}+B_{111} S_{r r} E_{r}+B_{122} S_{\theta \theta} E_{r} \\
& +B_{423} S_{z z} E_{r}+B_{133} S_{r z} E_{z}, \\
D_{z}= & e_{31} S_{r r}+e_{31} S_{\theta \theta}+e_{33} S_{z z}+\epsilon_{33}^{S} E_{z} \\
& +\left(B_{133} S_{r r}+B_{423} S_{\theta \theta}+B_{333} S_{z z}\right) E_{z} \\
& +\frac{\left(B_{111}-B_{122}\right)}{2} S_{r z} E_{r}+P^{\mathrm{sp}} .
\end{aligned}
$$

In a wurtzite GaN/AlN crystal the tensorial coefficients in equations (5) take numerical values given in table 1 according to the following references $[55,56,19]$. First, we confirm that the normal strain component $\epsilon_{z z}$ is completely symmetric in the
Table 1. Stiffness coefficients, lattice constants, piezoelectric constants and permittivity coefficients are given relative to the vacuum permittivity.

\begin{tabular}{lcc}
\hline Parameter & GaN & AlN \\
\hline$c_{11}^{E}(\mathrm{GPa})$ & 390 & 396 \\
$c_{12}^{E}(\mathrm{GPa})$ & 145 & 137 \\
$c_{13}^{E}(\mathrm{GPa})$ & 106 & 108 \\
$c_{33}^{E}(\mathrm{GPa})$ & 398 & 373 \\
$c_{44}^{E}(\mathrm{GPa})$ & 105 & 116 \\
$a(\AA)$ & 3.189 & 3.112 \\
$c(\AA)$ & 5.185 & 4.982 \\
$\left.e_{15}(\mathrm{C} \mathrm{m})^{2}\right)$ & -0.49 & -0.60 \\
$e_{31}\left(\mathrm{C} \mathrm{m}^{2}\right)$ & -0.49 & -0.60 \\
$e_{33}\left(\mathrm{C} \mathrm{m}^{2}\right)$ & 0.73 & 1.46 \\
$\epsilon_{\|}^{S}=\epsilon_{33}^{S}$ & 10.01 & 8.57 \\
$\epsilon_{\perp}^{S}=\epsilon_{11}^{S}=\epsilon_{22}^{S}$ & 9.28 & 8.67 \\
$P^{\mathrm{sp}}\left(\mathrm{C} \mathrm{m}^{2}\right)$ & -0.029 & -0.081 \\
\hline
\end{tabular}

case where the GaN/AlN quantum dot is considered without a wetting layer (see figure 1(a)), as expected from previous studies [57].

\section{Results and discussions}

Since the electrostrictive coefficient $\left(B_{333}\right)$ that enters the model appears in equations for $T_{z z}$ and $D_{z}$ only, there is no influence on the normal strain in the $r$ direction $\left(\epsilon_{r r}\right)$. The normal strain in the $z$ direction $\left(\epsilon_{z z}\right)$ for our GaN/AlN quantum dot with a wetting layer is presented in figures 1(b) and (c). The distribution of dielectric potential in this case is given in figure 2(a). We note that the linear results presented in figures 1 and 2(a) for the quantum dots with a wetting layer are in agreement with the results of $[57,58]$. We observe that the major influence of the electrostrictive term is inside the quantum dot $(\mathrm{GaN})$ although there are minor deviations in the matrix material (AlN) too. As expected, the linear results of $\epsilon_{z z}$ and the results from the nonlinear case with the lowest value of $M_{33}=10^{-21} \mathrm{~m}^{2} \mathrm{~V}^{-2}$ are almost identical, since the nonlinear effect has been reduced by decreasing the electrostrictive coefficient. As shown in figure 1(b), $\epsilon_{z z}$ is changing to a flat line by increasing the electrostrictive coefficient $M_{33}$ which is caused by a dramatic change in the electric potential from the linear case presented in figure 2(a) to the nonlinear case in figure 2(b). As seen from figure 2, the maximum and minimum voltages have dropped dramatically from $1.761 \mathrm{~V}$ to $0.255 \mathrm{~V}$ and from $-1.437 \mathrm{~V}$ to $-0.283 \mathrm{~V}$, respectively, caused by the higher-order effect which moves the pick points to the side edges and recovers the voltage on the top and bottom edges. However, the order of magnitude of the electric field $\left(10^{7} \mathrm{MV} \mathrm{cm}^{-1}\right)$ remains the same as reported [59].

In order to highlight the influence of nonlinear electromechanical effects, and particularly electrostriction on optoelectronic properties, we analyze the band structure of our quantum dots with an eight-band $\mathbf{k} \cdot \mathbf{p}$ model [2]. Here we consider an experimentally reported value of $M_{33}=1.2 \times$ 


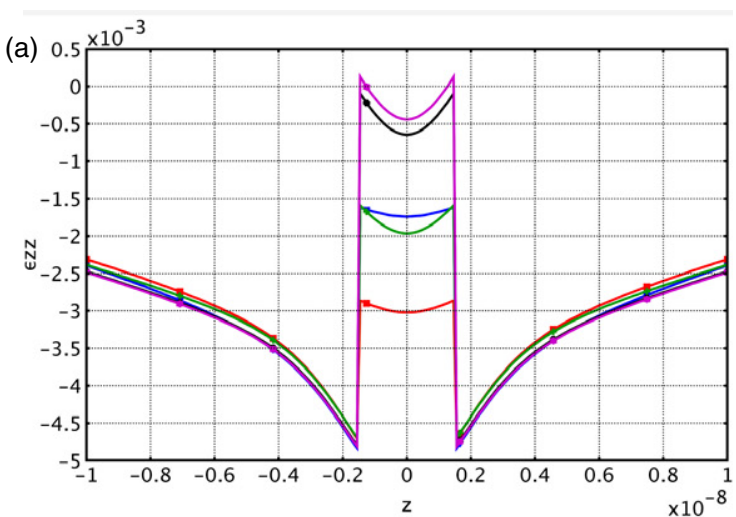

(b)

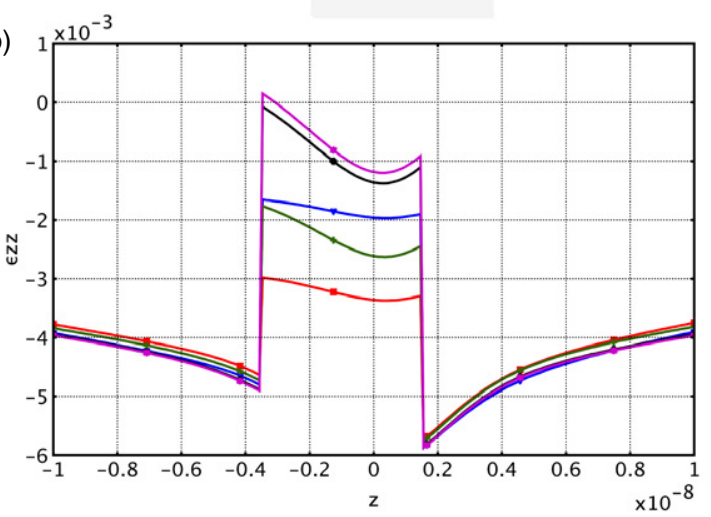

(c)

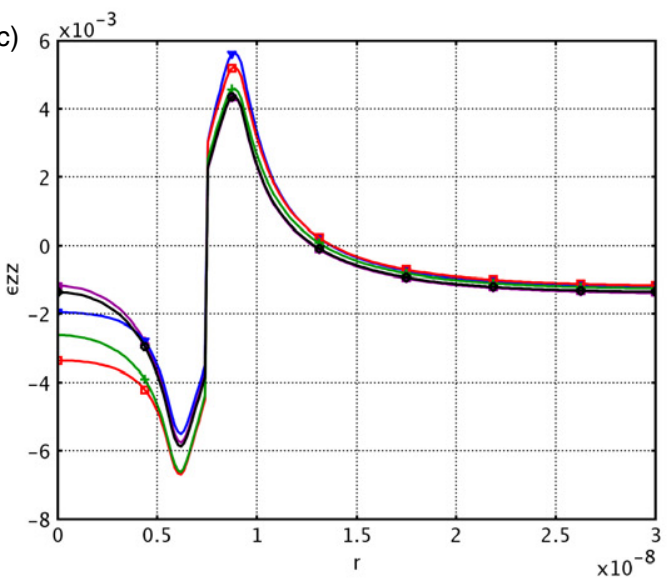

Figure 1. Strain in $z$ direction $\left(\epsilon_{z z}\right)$. The linear case is shown with magenta line, star marker, and the nonlinear case is shown as follows: $M_{33}=10^{-18} \mathrm{~m}^{2} \mathrm{~V}^{-2}$ (blue line, triangle marker), $10^{-19} \mathrm{~m}^{2} \mathrm{~V}^{-2}$ (red line, square marker), $10^{-20} \mathrm{~m}^{2} \mathrm{~V}^{-2}$ (green line, plus marker) and $10^{-21} \mathrm{~m}^{2} \mathrm{~V}^{-2}$ (black line, circle marker). (a) Normal strain in $z$ direction $\left(\epsilon_{z z}\right)$ on a vertical axial line in $z$ direction $(r=0)$ for the cylindrical GaN/AIN quantum dot without wetting layer. (b) Normal strain in $z$ direction $\left(\epsilon_{z z}\right)$ on a vertical axial line in $z$ direction $(r=0)$ for the conical GaN/AlN quantum dot with wetting layer. (c) Normal strain in $z$ direction $\left(\epsilon_{z z}\right)$ on a horizontal radial line in $r$ direction $(z=0)$ for the conical GaN/AlN quantum dot with wetting layer.

$10^{-18} \mathrm{~m}^{2} \mathrm{~V}^{-2}$. We consider two conduction bands coupled with six valence bands including heavy-hole, light-hole and spin-orbit bands. The crystal-field splitting is also accounted for. The unstrained valence band edge of AlN is taken as a reference. Details of the expressions and parameters for
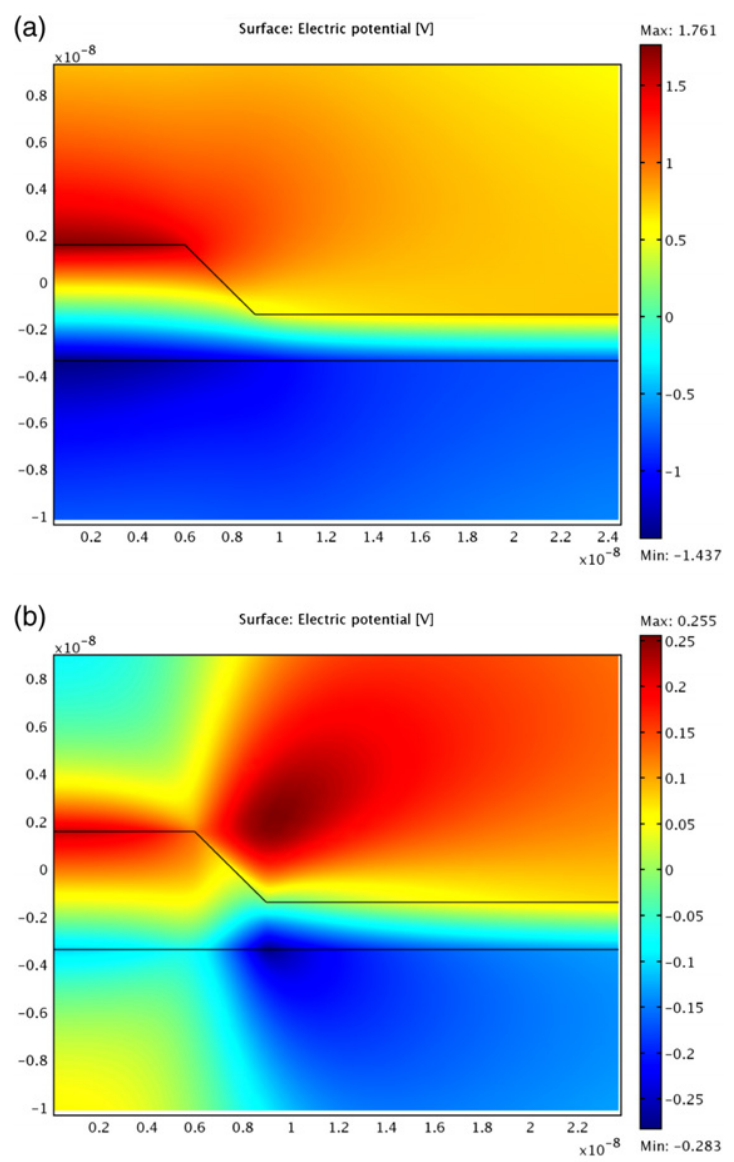

Figure 2. Cross-sectional $(r-z)$ distribution of electric potential. (a) Linear case that neglects the electrostriction effect. (b) Nonlinear case accounting for the electrostriction effect.

the eight-band $\mathbf{k} \cdot \mathbf{p}$ Hamiltonian can be found in [60-62]. The wavefunctions for the ground state of truncated conical GaN/AlN QDs with WL have been calculated using an eightband $\mathbf{k} \cdot \mathbf{p}$ model. They are presented in figure 3, demonstrating the influence of the piezoelectric and electrostriction effects on electronic localization and ground state energy for the truncated conical QD with WL of thickness $2 \mathrm{~nm}$ and with a height of $3 \mathrm{~nm}$ and radius of $6 \mathrm{~nm}$. When neither of the effects, piezoelectric or electrostrictive, is accounted for, figure 3(a) shows that the electron localization is symmetric with a ground state energy of $4.1449 \mathrm{eV}$. However, the upward shift in localization and a significant decrease in the ground state energy, $2.627926 \mathrm{eV}$, is observed when electromechanical effects are accounted for, as shown from figure 3(b). Further, on accounting for the electrostriction, the electronic localization as well as the ground state energy becomes similar to the case of figure 3(a) with a ground state energy of $4.417723 \mathrm{eV}$. We also observe a similar trend for other geometries, for example cylindrical QDs with the ground state energies, $4.19713 \mathrm{eV}, 3.453402 \mathrm{eV}$ and $3.763699 \mathrm{eV}$, for the cases without piezoelectricity and electrostriction, with piezoelectricity, and with piezoelectricity and electrostriction, respectively.

The electrons are pushed to the QD top when piezoelectric effects are taken into account. This is due to the significantly 

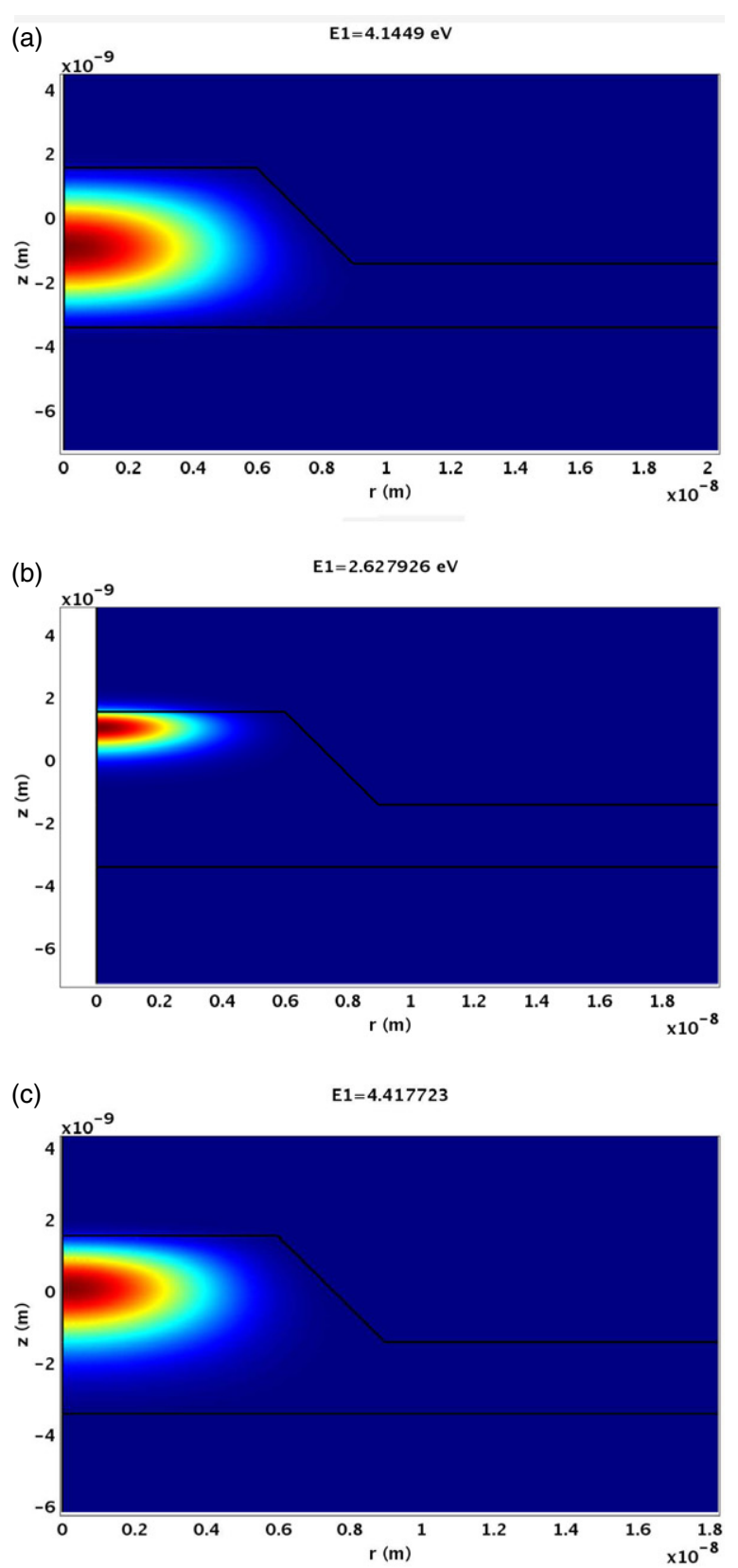

Figure 3. Influence of linear and nonlinear electromechanical properties on band structure of the QD with WL. (a) Without piezoelectricity and electrostriction. (b) With piezoelectricity. (c) With piezoelectricity and electrostriction.

higher magnitudes of the electric potential and the nature of its profile (figures 2(a) and (b)) [60-63]. This effect was experimentally observed through magneto-tunneling spectroscopy measurements that allow the mapping of the electron wavefunctions [64] and was also theoretically proved [65]. The reorganization of electron localization along with the ground state energy is a result of the linear electromechanical phenomena as well as the electrostriction which have the opposite effect on the resulting electromechanical fields and subsequently on band structures [28]. These findings lead to several new questions if one attempts to compare them with the previous experimental results $[59,60]$, where a dramatic decrease of the emission energy with the QD height has been observed. To answer such questions, the effect of all electrostriction coefficients should be analyzed. However, experimental results on such coefficients are currently absent in the literature.

\section{Conclusions}

In summary, we have analyzed the effect of nonlinear electrostriction on electromechanical properties of quantum dots. In particular, we have demonstrated that the contribution of higher-order nonlinear electrostrictive terms can be significant, leading to new insights into physical properties of low-dimensional nanostructures. The importance of these new results has been exemplified by band structure calculations based on a multiband model. Further experimental studies of electrostrictive coefficients in $\mathrm{GaN}$ are necessary for a more precise evaluation of the contribution of such nonlinear terms.

\section{Acknowledgments}

This work, conducted in the $\mathrm{M}^{2} \mathrm{NeT}$ Laboratory (http://www. m2netlab.wlu.ca), was made possible by the facilities of the SHARCNET. RM and SP acknowledge the support from the NSERC and CRC program, Canada.

\section{References}

[1] Piprek J 2007 Nitride Semiconductor Devices: Principles and Simulation (New York: Wiley-VCH)

[2] Patil S R and Melnik R V N 2009 Nanotechnology 20125402

[3] Patil S R and Melnik R V N 2009 Phys. Status Solidi a 206 960-4

[4] Patil S, Hong W P and Park S H 2008 Phys. Lett. A 372 4076-9

[5] Patil S, Wen B and Melnik R V N 2009 Strain effects and temperature-dependent phase stability of II-VI semiconductor nanostructures Physics of Semiconductors: 29th Int. Conf. on Physics of Semiconductors (Rio de Janeiro); AIP Conf. Proc. 1199 303-4

[6] Mahapatra D R and Melnik R V N 2007 Analysis of low-dimensional semiconductor nanostructures with a self-consistent iterative scheme Micro- and Nanotechnology: Materials, Processes, Packaging, and Systems III (Adelaide); Proc. SPIE 6415 A4150

[7] Melnik R V N, Lassen B, Lew Yan Voon L C, Willatzen M and Galeriu C 2005 Nonlinear Anal. 63 2165-76

[8] Lassen B, Willatzen M and Melnik R 2006 J. Comput. Theor. Nanosci. 3 588-97

[9] Melnik R and Mahapatra R 2007 Comput. Struct. 85 11-4

[10] Mahapatra D R, Sutrakar V K and Melnik R V N 2009 Temperature and stress controlled surface manipulation in Ni-Al nano-layers Nanotech 2009 Conf.: Nanotech Conf. \& Expo 2009, Technical Proc.-Nanotechnology: Biofuels, Renewable Energy, Coating Fluidics and Compact Modeling (Houston) vol 3, ed M Laudon and

B Romanowicz pp 227-30

[11] Melnik R and Wang L 2010 Phase-field approach to studying shape memory effects and thermomechanical properties of low dimensional nanostructures Advanced Research in Physics and Engineering: 2nd WSEAS International Conf. on Nanotechnology (Cambridge, England) (Mathematics and Computers in Science and Engineering) ed O Martin et al pp 125-30 
[12] Mahapatra D R and Melnik R 2006 Modeling the essential atomistic influence in the phase transformation dynamics of shape memory materials Smart Structures and Materials 2006: Active Materilas: Behavior and Mechanics; Proc. SPIE 6170 61700Y

[13] Melnik R, Tsviliuk O and Wang L 2009 Low dimensional nonlinear thermomechanical models describing phase transformations and their applications Recent Advances in Applied Mathematics (14th WSEAS Int. Conf. on Applied Mathematics) (Mathematics and Computers in Science and Engineering) ed C A Bulucea et al pp 83-8

[14] Wang L and Melnik R V N $2010 \mathrm{~J}$. Appl. Mech.-Trans. ASME 77031015

[15] Mahapatra R D, Melnik R V N, Willatzen M, Lassen B and Voon L L Y 2006 Poisson-Schrodinger model in the analysis of coupled effects in quantum well nanostructures 2006 Conf. on Optoelectronic and Microelectronic Materials \& Devices (Perth, Australia) (Piscataway, NJ: IEEE) pp 305-7

[16] Patil S et al 2009 Thermopiezoelectric and nonlinear electromechanical effects in quantum dots and nanowires Physics of Semiconductors; 29th Int. Conf. on Physics of Semiconductors (Rio de Janeiro); AIP Conf. Proc. $1199327-8$

[17] Willatzen M, Lassen B, Lew Yan Voon L C and Melnik R V N 2006 J. Appl. Phys. 100024302

[18] Guy I L, Muensit S and Goldys E M 1999 Appl. Phys. Lett. 753641

[19] Fonoberov V A and Balandin A A 2004 J. Vac. Sci. Technol. B 222190

[20] Fonoberov V A and Balandin A A 2004 Phys. Status Solidi c $12650-3$

[21] Fonoberov V A and Balandin A A 2004 Phys. Rev. B 70233205

[22] Patil S R and Melnik R V N 2009 J. Phys. D: Appl. Phys. 42145113

[23] Patil S R and Melnik R V N 2009 Proc. Eng. 1 105-8

[24] Willatzen M, Wang L and Voon L C L Y 2008 Superlatt. Microstruct. 43 436-40

[25] Vaschenko G, Patel D, Menoni C S, Ng H M and Cho A Y 2002 Appl. Phys. Lett. 804211

[26] Yan W S, Zhang R, Xiu X Q, Xie Z L, Han P, Jiang R L, Gu S L, Shi Y and Zheng Y D 2007 Appl. Phys. Lett. 90182113

[27] Yan W S, Zhang R, Xie Z L, Xiu X Q, Han P, Lu H, Chen P, Gu S L, Shi Y, Zheng Y D and Liu Z G 2009 Appl. Phys. Lett. 94042106

[28] Bester G, Wu X, Vanderbilt D and Zunger A 2006 Phys. Rev. Lett. 96187602

[29] El-Hami K and Matsushige K 2005 Ultramicroscopy 105 143-7

[30] Tang C, Guo W and Guo Y 2006 Appl. Phys. Lett. 88243112

[31] Mahapatra D R et al 2009 Mol. Simul. 35 512-9

[32] Kholkin A L et al 2001 J. Appl. Phys. 89 8066-73

[33] Mahapatra D R and Melnik R V N 2006 Mesoscopic dynamics of piezoelectric copolymer thin films Proc. SPIE $616861682 D$

[34] Turik A V, Yesis A A and Rexnitchenko L A 2006 J. Phys.: Condens. Matter 18 4839-43

[35] Even J, Dore F, Cornet C, Pedesseau L, Schliwa A and Bimberg D 2007 Appl. Phys. Lett. 91122112

[36] Bester G, Zunger A, Wu X and Vanderbilt D 2006 Phys. Rev. B 74081305
[37] Schliwa A, Winkelnkemper M and Bimberg D 2007 Phys. Rev. B 76205324

[38] Melnik R V N and Willatzen M 2002 Modelling coupled motion of electrons in quantum dots with wetting layers ICCN 2002: 2nd Int. Conf. on Computational Nanoscience and Nanotechnology (San Juan, PR) ed M Laudon and B Romanowicz, pp 209-12

[39] Melnik R V N and Zotsenko K N 2003 Computations of coupled electronic states in quantum dot/wetting layer cylindrical structures Lecture Notes in Computer Science: Computational Science-ICCS 2003, PT III, Proc. (Melbourne) ed P M A Sloot et al pp 343-9

[40] Melnik R V N and Zotsenko K N 2004 Modelling Simul. Mater. Sci. Eng. 12 465-77

[41] Melnik R V N and Willatzen M 2004 Nanotechnology 151

[42] Yang J 2005 An Introduction to the Theory of Piezoelectricity (Berlin: Springer)

[43] Vaschenko G, Menoni C S, Patel D, Tome C N, Clausen B, Gardner N F, Sun J, Goetz W, Ng H M and Cho A Y 2003 Phys. Status Solidi b 235238

[44] Joshi S P 1992 Smart Mater. Struct. 18043

[45] Melnik R V N 2000 Appl. Math. Comput. 107 27-55

[46] Melnik V N and Moskalkov M N 1988 USSR Comput. Math. Math. Phys. 28 109-10

[47] Melnik V N 1991 Izv. Vyssh. Uchebn. Zaved. Mat. 4 24-32

[48] Melnik V N 1997 Intelligent structures and coupling in mathematical models: examples from dynamic electroelasticity Proc. 5th Int. Properties and Applications of Dielectric Materials vols 1 and 2 (Piscataway, NJ: IEEE) pp 995-8

[49] Melnik R V N 1997 Math. Mechan. Solids 2 153-80

[50] Melnik R V N and Melnik K N 1998 Commun. Numer. Methods Eng. 14 839-47

[51] Melnik V N and Moskalkov M N 1991 Diff. Eqns 27 860-7

[52] Melnik R V N 1998 J. Diff. Eqns Appl. 4 185-212

[53] Melnik R V N and Melnik K N 2000 Appl. Math. Modelling 24 147-63

[54] Glavin B A et al 2005 Phys. Rev. B 71081305

[55] Adachi S 2004 Handbook on Physical Properties of Semiconductors (Boston, MA: Kluwer Academic)

[56] Christmas U M E, Andreev A D and Faux D A 2005 J. Appl. Phys. 98073522

[57] Lassen B, Willatzen M, Barettin D, Melnik R V N and Lew Yan Voon L C 2008 J. Phys.: Conf. Ser. 107012008

[58] Wang J and Chu H J 2006 J. Appl. Phys. 100053520

[59] Simon J, Pelekanos N T, Adelmann C, Martinez-Guerrero E, Andr R, Daudin B, Dang L S and Mariette H 2003 Phys. Rev. B 68035312

[60] Fonoberov V A and Balandin A A 2003 J. Appl. Phys. $947178-86$

[61] Nakaoka T, Kako S and Arakawa Y 2006 Physica E 32 148-51

[62] Lassen B, Willatzen M, Melnik R and Voon L C L Y 2006 J. Mater. Res. 21 2927-35

[63] Williams D P, Andreev A D, O'Reilly E P and Faux D A 2005 Phys. Rev. B 72235318

[64] Patane A et et al 2002 Phys. Rev. B 65165308

[65] Migliorato M A, Powell D, Liew S L, Cullis A G, Navaretti P, Steer M J, Hopkinson M, Fearn M and Jefferson J H 2004 J. Appl. Phys. 96 5169-72 